\title{
Development of Fast Atom Bombardment Mass Spectral Methods for the Identification of Carcinogen-Nucleoside Adducts
}

\author{
M. S. Bryant* and J. O. Lay, Jr. \\ Division of Biochemical Toxicology, National Center for Toxicological Research, Jefferson, Arkansas, USA
}

M. P. Chiarelli

Department of Chemistry, University of Arkansas at Little Rock, Little Rock, Arkansas, USA

\begin{abstract}
An analytical strategy using fast atom bombardment (FAB) ionization and tandem mass spectrometry has been developed to determine the molecular weight and major fragment ions, and to provide limited structural characterization of low picomole levels of carcinogen-nucleoside adducts. This strategy consists of three main components: (1) the sensitivity for analysis by $\mathrm{FAB}$ combined with mass spectrometry is increased via chemical derivatization; (2) the nucleoside adducts are selectively detected by using constant neutral loss scans; and (3) structurally characteristic fragments are obtained by using daughter ion scans. Trimethylsilyl derivatized arylamine-nucleoside adducts have been detected at levels as low as a few picomoles by using this approach. After experimental determination of the mass of the $\mathrm{BH}_{2}^{+}$fragment ion, daughter ion spectra have been used to probe the structure specificity associated with collision-activated decomposition of this fragment. With model C-8 substituted arylamine adducts [ $\mathrm{N}$-(deoxyguanosin 8 yl)-4-aminobiphenyl, $\mathrm{N}$-(deoxyadenosin-8-yl)-4-aminobiphenyl, and $\mathrm{N}$-(deoxyguanosin-8-yl)-2-aminofluorene], nucleoside-specific and carcinogen-specific fragmentation have been observed in daughter ion spectra. (T Am Soc Mass Spectrom 1992, 3, 360-371)
\end{abstract}

$\mathrm{A}$ central tenet of cancer research is that covalent carcinogen-DNA adducts are associated with the risk of developing tumors [1]. Not surprisingly, it has been suggested that these adducts could be used as biological dosimeters of exposure to chemical carcinogens. This approach offers significant advantages over traditional methods of risk assessment, such as environmental monitoring [2 7]. This use of carcinogen-DNA adducts, however, is critically dependent upon the development of aralytical methods that allow their identification and quantification at very low (picomole) levels [1].

Methods to measure DNA adducts in human tissues have employed enzyme immunoassays [8-13], fluorescence assays $[11,14]$, and ${ }^{32} \mathrm{P}$-postlabeling $[8$, 15-17]. The principal advantage of these techniques is their great sensitivity. For example, the ${ }^{32} \mathrm{P}$-postlabeling method has the capability to detect minute quantities of adducts, perhaps one adduct in $10^{10} \mathrm{nu}-$

\footnotetext{
*Current address: Chemical Industry Institute for Toxicology, Research Triangle Park, NC 27709.

Address reprint requests to Jackson O. I ay, Jr., Division of Bio chemical Toxicology, National Center for Toxicological Research, Jeffersur, AR 72079.
}

cleotides [18]. Nevertheless, identifications using this method are generally based on cochromatography with known standards, and at best, only tentative identifications can be made for unknown adducts. The other widely used technique, immunoassay, involves the use of antibodies raised against either the specific carcinogen-modifted DNA or nucleoside adducts coupled to a protein carrier [19]. Methods based on immunoassay suffer from some limitations when applied to the identification of unknowns. False-positive confirmations may arise due to cross reactivity with structurally similar adducts. Also, unknown adducts will likely not elicit a response to a specific antibody derived from a carcinogen of unrelated structure, and thus remain undetected. This combination of exquisite sensitivity and poor structure specificity has resulted in the isolation of carcinugen-DNA adducts that can be detected but not identified (see ref 8 for example).

Electron impact mass spectrometry (EI/MS) has the sensitivity to detect comparably low levels of volatile organic compounds. This technique has not been successfully applied to the analysis of carcinogen-modified deoxynucleosides isolated from tissue DNA, even 
when the compounds are derivatized to increase volatility. Chemical ionization mass spectral methods for the detection of specific DNA adducts have been reported, especially with electrophore labels to enhance negative ion formation [20-22]. Typically, the sugar is removed to enhance volatility prior to electrophore derivatization. Giese and co-workers [20, 21] accomplished this using sequential oxidation and reduction prior to derivatization. Similarly, Swenberg and co-workers [22] have used gas chromatography/ mass spectrometry (GC/MS) with negative ion chemical ionization (NICI) to analyze trace levels of $\mathrm{N}^{2}-3-$ ethenoguanine following electrophore derivatization.

Fast atom bombardment (FAB) ionization has been shown amenable to the identification of microgram levels of purified nucleosides [23-25] and carcinogennucleoside/nucleotide adducts [26]. We have reported the identification of deoxynucleoside/nucleotide adducts based, in part, on analysis by FAB combined with mass spectrometry (FAB/MS) [27-30]. Unfortunately, for applications involving the analysis of tissue samples, microgram quantities of adducts cannot easily be obtained. Thus, we have become interested in methods that allow the identification of unknown carcinogen-deoxynucleoside adducts at levels in the $1-10 \mathrm{ng}$ range.

Trimethylsilylated derivatives of deoxynucleosidecarcinogen adducts show surprising sensitivity by FAB/MS, possibly even grcater than EI/MS $[27,31]$. For example, the adduct reported in ref 27, $\mathrm{N}$-(deoxyadenosin-8-yl)-4-amino-3-chlorobenzyl alcohol, was correctly identified after FAB/MS analysis of the silylated derivative but not by using EI/MS. Recent results from Schram's laboratory $[32,33]$ show surprisingly low level detection for silylated derivatives of related compounds, including ribonucleosides and ribonucleotide monophosphate derivatives by FAB/MS. This approach is analogous to the electrophore derivatization methods used to enhance the detection of alkylated nucleosides and bases using NICI mass spectrometry [34], or electron capture GC [35].

In this article we report the development of FAB combined with tandem mass spectrometry methodologies for the analysis of derivatized arylamine-deoxynucleoside adducts at levels as low as $1 \mathrm{ng}$. The adducts are converted to trimethylsilyl (TMS) derivatives and examined by using FAB ionization and constant neutral loss (CNL) scans. FAB ionization provides a characteristic mass spectral fragment-marker ion (sugar loss) that can be used in CNL scans to search for potential nucleoside adducts. Subsequent daughter ion scans, using the experimentally determined $\mathrm{BH}_{2}^{+}$fragment ion rather than the protonated (or deprotonated) molecule ion, are then used to provide evidence for adduct structures. This method is applicable even when the mass selected parent ion cannot be identified/recognized in the normal FAB mass spectrum. Results obtained by using underiva- tized adducts and soft ionization methods other than FAB/MS are reported for comparison. Preliminary accounts of our work have been presented [36-38].

\section{Experimental}

Adduct synthesis. N-(2'-deoxyguanosin-8-yl)-4aminobiphenyl was synthesized by a modification of a published procedure [39] and then purified by preparative high-performance liquid chromatography (HPLC). N-(guan-8-yl)-4-aminobiphenyl and N-(guan8-yl)-4-aminobiphenyl- $d_{9}$ were synthesized by reaction of 4-aminobiphenyl or 4-aminobiphenyl$2,2^{\prime}, 3,3^{\prime}, 4,5,5^{\prime}, 6,6^{\prime}-\mathrm{d}_{9}$ with guanine-N-oxide in $10: 1$ dimethyl sulfoxide/dimethylformamide at room temperature overnight. The other adducts were obtained from Frederick A. Beland and Fred F. Kadlubar of the National Center for Toxicological Research.

Derivatization procedure A. Measured portions of the adduct were added to tapered glass vials and the ethanol solvent was removed with dry argon gas. The silylating reagent (N-methyl-N-trimethyl-silyltrifluoroacetamide [MSTFA], N,O-bis(trimethylsilyl)trifluoro-acetamide [BSTFA], or N-methyl-N-(tertbutyldimethylsilyl)-trifluoroacetamide [MTBSTFA]) in acetonitrile or dimethylformamide $(1: 1 \mathrm{v} / \mathrm{v})$ was then added under argon and allowed to react 1-2 h at room temperature or at $60-100{ }^{\circ} \mathrm{C}$ for $1 \mathrm{~h}$.

Derioatization procedure B. Adducts were silylated by adding the reagent directly to the dry sample and heating at $60-100{ }^{\circ} \mathrm{C}$ for $1-2 \mathrm{~h}$. Mass spectral analyses were performed directly on the resulting solutions containing the derivatized sample dissolved in the derivatizing reagent.

Thermospray mass spectrometry (TS/MS). TS mass spectra were obtained by using a Finnigan MAT (San Jose, CA) TSQ-70 triple stage quadrupole mass spectrometer equipped with a Finnigan TS controller and source. An ISCO (Lincoln, NE) LC-5000 syringe pump connected to a Rheodyne 7125 injector $(20 \mu \mathrm{L}$ loop) provided a flow rate of $-1.5 \mathrm{~mL} / \mathrm{min}$. The mobile phase typically was $80-90 \%$ aqueous buffer $(0.1 \mathrm{M}$ ammonium acetate and methanol). The vaporizer temperature was adjusted to a cool setting $\left(\sim 90^{\circ} \mathrm{C}\right)$ to maximize abundance of the protonated molecule.

Desorption chemical ionization (DCI) mass spectra. DCI mass spectra were obtained with a Finnigan MAT 4023 operating in the $\mathrm{CI}$ mode with methane or $10 \%$ ammonia in nitrogen as the reagent gas. Samples were introduced via a Vacumetrics (Ventura, CA) or a custom-made $\mathrm{DCI}$ probe incorporating a platinum filament heated at a rate of $50 \mathrm{~mA} / \mathrm{s}$ for $60 \mathrm{~s}$. The reagent gas was set to an uncorrected nominal source pressure of 0.25 torr. The source temperature was $200-220{ }^{\circ} \mathrm{C}$. 
$F A B$ mass spectrometry. Samples were applied directly to a copper target via a syringe $\left(0.5-1.0{ }_{\mu} \mathrm{L}\right.$. .) after application of $1-2 \mu \mathrm{L}$ of thioglycerol FAB matrix liquid. Spectra were obtained by using a Finnigan MAT TSQ-70 with an Ion Tech (Middlesex, UK) gun. FAB ionization was effected by using xenon atoms accelerated to $8-10 \mathrm{keV}$. Scans were obtained from about $900 \mathrm{u}$ down to about $70 \mathrm{u}$ under control of the TSQ-70 data system.

FAB/MS/MS. (a) Constant neutral loss scans were acquired by using the Finnigan MAT TSQ 70 as described above for FAB/MS. To obtain a constant neutral loss scan the first and last quadrupoles, Q1 and Q3, were scanned at the same rate with a constant mass offset corresponding either to the mass of a deoxyribose moiety (116 u) or a bis-TMS derivatized deoxyribose (260 u). (b) Daughter ion scans were obtained by fuxing the mass passed by Q1 to correspond to the protonated molecule, the $\mathrm{BH}_{2}^{+}$ion, or a TMS derivative of the former, while the mass of Q3 was scanned to detect the masses of all fragments formed by decomposition of the mass selected parent ion. Both types of scans were acquired by using argon as the collision gas to induce decomposition of the parent ion. Collision energies of $30-90 \mathrm{eV}$ were utilized and collision cell pressures were varied to maximize the yield of useful daughter ions.

\section{Results and Discussion}

\section{Sensitivity Studies}

The initial objectives of the sensitivity studies were: (a) to demonstrate that FAB/MS is a sensitive soft ionization methodology for the analysis of carcinogen-modified deoxynucleosides; and (b) to optimize experimental conditions so that full-scan spectra could be obtained by using minimum amounts of sample. The analysis of carcinogen-DNA adducts from in vivo rather than in vitro studies is currently hindered by the inability to routinely obtain mass spectra from low nanogram to picogram levels of samples.

Comparison of ionization methods. To address the furst objective, mass spectra of the model adduct $\mathrm{N}$-(deoxyguanosin-8-yl)-4-aminobiphenyl (dG-C8-ABP, Figure 1) were obtained by using DCI, TS, and FAB ionization. [This adduct, the principal modified nucleoside isolated from urinary bladder DNA upon exposure to the arylamine carcinogen 4-aminobiphenyl $(A B P)$, is attributed to a reaction between DNA and a reactive metabolite, namely N-hydroxy-ABP.] Analyses were attempted by using $1 \mu \mathrm{g}, 500 \mathrm{ng}, 200 \mathrm{ng}, 100$ $\mathrm{ng}$, and $10 \mathrm{ng}$ of adduct with each of the ionization techniques. Typically, spectra were obtained by scanning from $600 \mathrm{u}$ down to $100 \mathrm{~m}$. The nominal sample sizes giving reproducible full-scan spectra under the conditions described above are enumerated in Table<smiles>Nc1nc2c(nc(Nc3ccc(-c4ccccc4)cc3)n2[Tl])c(=O)[nH]1</smiles><smiles>CCn1c(Nc2ccc3c(c2)Cc2ccccc2-3)nc2c1N=C(N)N(C(F)(F)F)NC2=O</smiles>

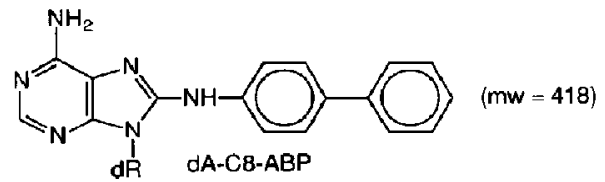<smiles>COC(=O)Nc1ccc2c(c1)Cc1ccccc1-2</smiles>

Figure 1. The structures of model nucleoside-carcinogen adducts used in this study: $\mathrm{N}$-(deoxyguanosin-8-yl)-4-aminobiphenyl, dG-C8-ABP; N-(deoxyguanosin-8-yl)-2-aminofluorene, dG-C8-AF; N-(deoxy-adenosin-8-yl)-4-aminobiphenyl. dA-C8$A B P$, and N-(deoxyguanosin-8-yl)-2-acetylaminofluorene, dGC8-AAF.

1. For each ionization technique, the lowest limit of detection was 100 or $200 \mathrm{ng}$. Figure 2 shows a typical spectrum obtained from the analysis of $100 \mathrm{ng}$ of dG-C8-ABP by using FAB ionization. The protonated molecule and $\mathrm{BH}_{2}^{+}$ion are detectable above the near continuous background signal attributed to the FAB matrix liquid (thioglycerol). Moser and Wood [24] and Weng et al. [32] have reported comparable detection limits (250-500 $\mathrm{ng}$ ) for unmodified nucleotides by FAB/MS using $p$-toluenesulfonic acid/glycerol or glycerol as matrix compounds.

For comparison the experiment was repeated by using $\mathrm{dG}-\mathrm{C} 8$-ABP derivatized to incorporate TMS groups. Solutions of the derivatizing reagent, a solvent, and the adduct were prepared so that the appropriate amounts of adduct ( $1 \mu \mathrm{g}$ to $10 \mathrm{ng}$ ) could be analyzed directly from the derivatization solution. TS/MS was not used in this comparison as the derivatized adducts were not expected to be stable in an aqueous solution. Procedure A was used for derivatization with MSTFA. Mass spectra were acquired over a wide mass range $(900 \mathrm{u})$ to simulate the experimental conditions appropriate for the examination of unknown adducts. For example, the molecular weight of the tetrakis-TMS derivative of a deoxyguanosine adduct of a carcinogen having a molecular weight above $350 \mathrm{u}$ would exceed $900 \mathrm{u}$. 
Table 1. Compilation of the minimum amount of carcinogen-nucleoside adduct needed to obtain full-scan mass spectra by using various soft ionization mass spectrometery methods

\begin{tabular}{|c|c|c|c|c|}
\hline Method & Adduct & Derivative $^{a}$ & Amount $^{b}$ & Figure \\
\hline $\mathrm{DCl} / \mathrm{MS}^{\circ}$ & dG-C8-ABP & - & $200 \mathrm{ng}$ & - \\
\hline $\mathrm{DCl} / \mathrm{MS}^{\mathrm{d}}$ & $d G-C B-A B P$ & - & $200 \mathrm{ng}$ & - \\
\hline TS/MS & dG-C8-ABP & 一 & $200 \mathrm{ng}$ & - \\
\hline $\mathrm{FAB} / \mathrm{MS}$ & dG-CB-ABP & - & $100 \mathrm{ng}$ & 2 \\
\hline $\mathrm{DCl} / \mathrm{MS}^{\mathrm{c}}$ & dG-C8-ABP & MSTFA (A) & $200 \mathrm{ng}$ & 3 \\
\hline $\mathrm{DCl} / \mathrm{MS}^{\mathrm{d}}$ & dG-C8-ABP & MSTFA (A) & $200 \mathrm{ng}$ & - \\
\hline FAB /MS & dG-C8-ABP & MSTFA (A) & $10 \mathrm{ng}$ & 4 \\
\hline $\mathrm{FAB} / \mathrm{MS}$ & dG-C8-AF & MSTFA (A) & $5 \mathrm{ng}$ & 5 \\
\hline FAB /MS & dG-CB-AF & MSTFA (B) & $1 \mathbf{n g}$ & - \\
\hline
\end{tabular}

Abbreviations: DCI = desorption chemical ionization;

$F A B=$ fast atom bombardment ionization;

MSTFA = N-methyl-N-trimethylsilyl-trifluoroacetamide;

ng = nanograms;

TS = thermospray ionizetion.

A and $B$ refer to the derivatization procedures (see Experimental).

Applied to the probe tip.

cMathane reagent gas.

dAmmonia reagent gas.

Incorporation of TMS groups did not significantly reduce the amount of adduct needed to produce spectra using DCI/MS with either methane or ammonia as the reagent gas. Typical of these results is the methane DCl mass spectrum shown in Figure 3. About $200 \mathrm{ng}$ of dG-C8-ABP derivatized with MSTFA/DMF was required to obtain reproducible spectra. While the protonated molecules for the tris- and tetrakis-TMS containing derivatives ( $m / z 651$ and 723) were observed, the fragment ions corresponding to TMS containing $\mathrm{BH}_{2}^{+}$ions $(m / z 391$ or 463$)$ were not. The ions at $m / z 487,413$, and 307 have not been identified.

In contrast to the DCI work, FAB mass spectra were obtained by using smaller amounts of silylated adduct. Figure 4 shows the FAB mass spectrum obtained when a 100-ng sample of dG-C8-ABP was derivatized (Procedure $\mathrm{A}$ ) and $10 \%$ (10 $\mathrm{ng}$ ) applied to the probe tip for analysis. The ions at $m / z 651$ and

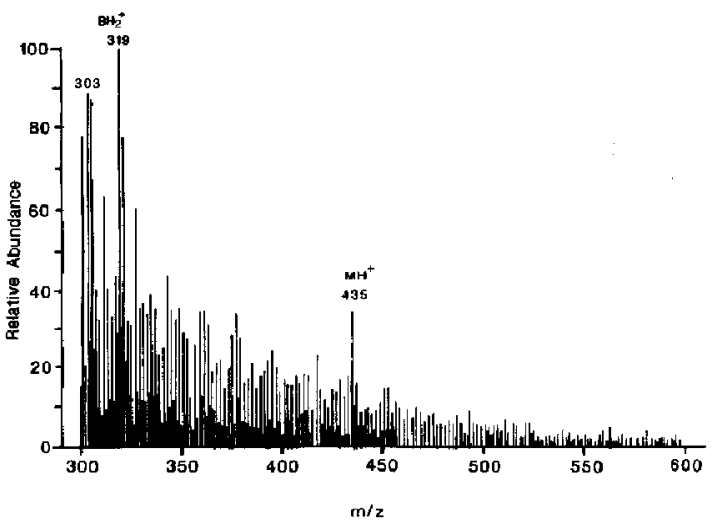

Figure 2. The FAB mass spectrum obtained by using $100 \mathrm{ng}$ of dG-C8-ABP showing the protonated molecule $(m / z 435)$ and $\mathrm{BH}_{2}^{+}$ion $(m / z 319)$.
579 correspond to the protonated molecules for the tris- and bis-TMS derivatives, whereas the ion at $\mathrm{m} / \mathrm{z}$ 391 corresponds to the mono-TMS derivative of the $\mathrm{BH}_{2}^{+}$fragment ion. This fragment could occur via loss of a bis-silylated deoxyribose from $m / z 651$ or via loss of a monosilylated sugar from $m / z 579$. Based on previous reports of complete silylation of the deoxyribose moiety, even with incomplete derivatization of the carcinogen/base moiety, the former pathway appears to be more likely [40]. The unsilylated fragment ion, $\mathrm{BH}_{2}^{+}$, was also observed at $m / z 319$ but is not displayed in Figure 4. This ion is attributed to loss of the disilylated deoxyribose moiety from those bissilylated adduct protonated molecules having both TMS groups attached to the sugar.

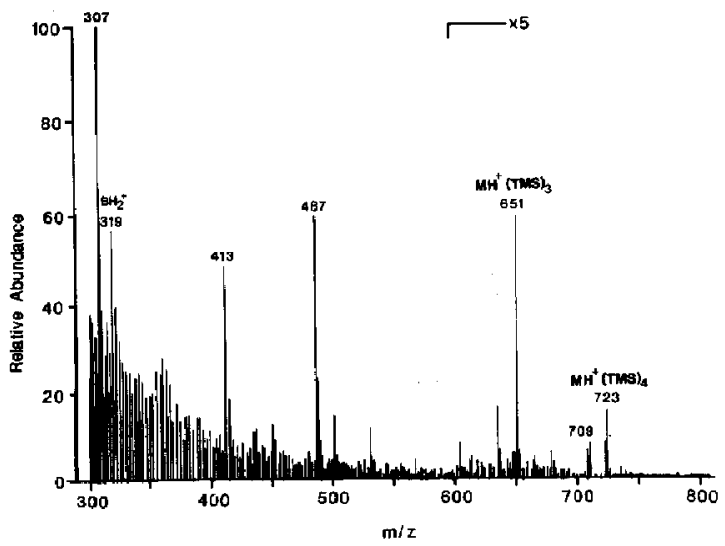

Figure 3. The methane DCI mass spectrum obtained by using $200 \mathrm{ng}$ of dG-C8-ABP derivatized with MSTFA/dimethylformamide showing protonated molecules for the tetrakis-TMS derivative $(m / z$ 723) and the tris-TMS derivative $(m / z 651)$, as well as a $\mathrm{BH}_{2}^{+}$ion with no TMS groups $(m / z 319)$. 


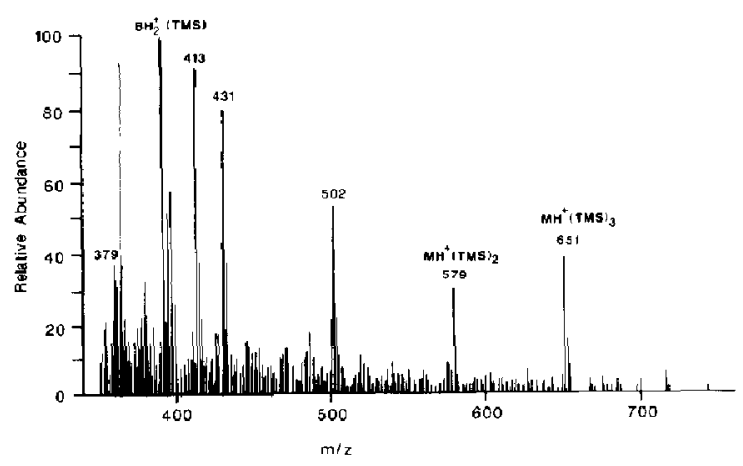

Figure 4. The FAB mass spectrum obtained by using $10 \mathrm{ng}$ of dG-C8-ABP derivatized with MSTFA/dimethylformamide showing protonated molecules for the tris-TMS $(m / z 651)$ and bis-TMS species $(m / z 579)$ and a $\mathrm{BH}_{2}^{+}$fragthent ion containing a single 'I'MS group $(m / z$ 341).

Optimization of derivatization conditions and comparison with other adducts. Similar experiments were also performed by using other adducts and other derivatization reagents. Figure 5 shows the spectrum obtained from $5 \mathrm{ng}$ of N-(deoxyguanosin-8-yl)-2aminofluorene, dG-C8-AF, derivatized with MSTFA (Procedure B). The ions at $m / z 663$ and 591 correspond to the tris- and bis-TMS containing protonated molecules, whereas $m / z 403$ and 331 are the $\mathrm{BH}_{2}^{+}$ fragments having one and no TMS groups, respectively. For comparison, the $m / z 217$ ion corresponds to the protonated thioglycerol matrix dimer. It should be noted that this matrix background ion is about 200 times smaller relative to the analyte protonated molecule ions using $5 \mathrm{ng}$ of derivatized adduct (Figure 5) than when using $1 \mu \mathrm{g}$ of underivatized adduct (Figure 6). This shows the striking improvement in

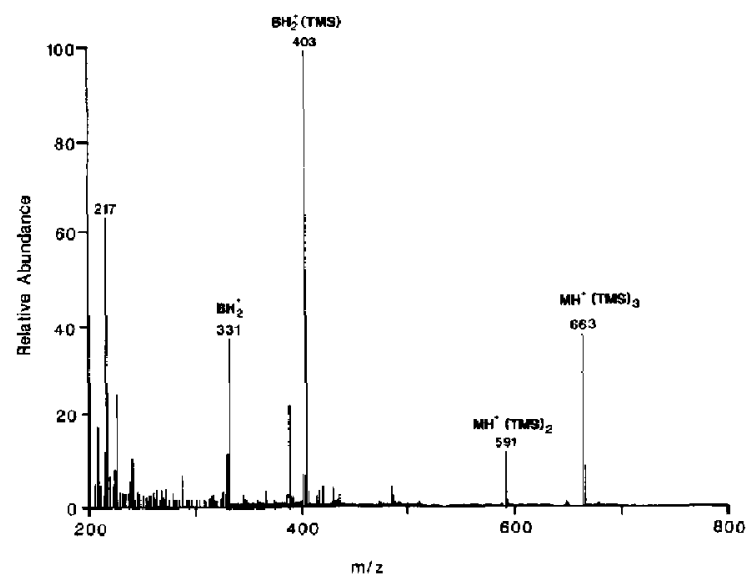

Figure 5. The FAB mass spectrum obtained by using $5 \mathrm{ng}$ of MSTFA derivatized $\mathrm{dG}-\mathrm{C}$ - $\mathrm{AF}$ showing protonated molecules for the tris- and bis-TMS containing ions ( $m / z 663$ and 591$)$ as well as the $\mathrm{BH}_{2}^{+}$TMS ion $(m / z 403)$ and the $\mathrm{BH}_{2}^{+}$ion $(m / z$ 331).

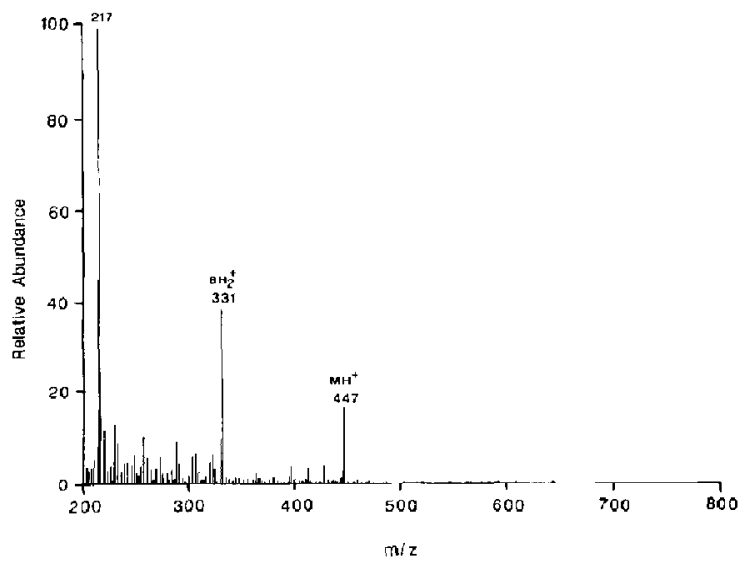

Figure 6. The FAB mass spectrum obtained by using $1 \mu \mathrm{g}$ of underivatized dG-C8-AF showing the protonated molecule $(m / z$ 447), the $\mathrm{BH}_{2}^{+}(m / z 331)$, and the relatively large contribution of the matrix ion at $m / z 217$.

the analyte to matrix signal levels observed with derivatized adducts as compared to their underivatized forms. The relative abundance of the analyte protonated molecules with respect to the thioglycerol matrix ion ( $m / z 217$ ) is even more surprising when the partitioning of the molecular signal among the two TMS derivatives ( $m / z 663$ and 591) is considered.

In a related study, Schram and co-workers [32] reported a method for the analysis of low nanogram levels of clinically important nucleotide-monophosphates by $F \wedge B / M S$. Derivatization was accomplished in capillary tubes by using small volumes of BSTFA in pyridine. Analysis by EI and $\mathrm{CI} / \mathrm{MS}$ indicated complete derivatization of the ribose monophosphate moiety, but not the base. They observed the loss of TMS groups, which they attributed to hydrolysis, by using either glycerol or 3-nitrobenzyl alcohol (NBA) as a FAB matrix liquid. One TMS group was lost with NBA, whereas more extensive hydrolysis and low signal levels were reported for glycerol. I Iydrolysis was not observed with tetraglyme, but other experimental difficulties, including lack of sensitivity, limiled its use as a FAB matrix. No results for thioglycerol were reported.

We also see evidence for hydrolysis with FAB/MS using thioglycerol. Differences in the number of TMS groups are observed when TMS derivatized adducts are analyzed by DCI/MS and FAB/MS (Figures 3 and 4, for example). However, the number of TMS groups appears constant from scan to scan via either method, suggesting that hydrolysis occurs rapidly and to completion prior to intruduction of the sample into the ion source. In other words, matrix induced hydrolysis likely occurs almost immediately upon introduction of the sample into the FAB matrix liquid.

If the DCI mass spectrum is assumed to accurately reflect the number of TMS groups added prior to introduction of the analyte into the $T A B$ matrix liquid, 
the number of groups expected by FAB/MS without hydrolysis would be $3-4$. The addition of this number of TMS groups, although it represents incomplete derivatization, is in good agreement with a previous study of carcinogen-nucleoside adducts using BSTFA and EI/MS [41]. The TMS derivatization of a similar C8-substituted deoxyguanosine adduct produced trisand tetrakis-TMS derivatives with a ratio of about 1:2. In our work we have typically observed bis- and tris-TMS derivatives in about the same ratio (i.e., Figures 4 and 5). These data suggest that only one TMS group is lost via hydrolysis in thioglycerol. This is a lesser extent of hydrolysis than was attributed to the use of glycerol in ref 32 , and is more consistent with the results reported for NBA. In other words, we attribute the observation of partially derivatized DNA-carcinogen adducts in thioglycerol to two causes. First, typical arylamine nucleoside adducts are not likely to be completely derivatized prior to their introduction into the matrix. Subsequently the thioglycerol matrix also induces loss of approximately one additional TMS group. The derivatization procedure may slightly affect the extent of this hydrolysis. When pure MSTFA is used for derivatization, the subsequent FAB spectra sometimes show a small increase in the tris-TMS derivative relative to the bis-TMS derivatized adduct. For example, the FAB/MS spectrum shown in Figure 4 was obtained by using 1:1 MSTFA/dimethylformamide, whereas Figure 5 represents derivatization in pure MSTFA. By either method, FAB/MS analysis of the TMS derivatives gives about the same detection limit in thioglycerol previously reported for NBA.

In another recent report, Vorous and co-workers [31] showed FAB/MS spectra of TMS derivatives of deoxynucleoside-carcinogen adducts. They reported extensive hydrolysis using thioglycerol, based on the presumption that the adducts were initially completely derivatized. However, they showed no evidence regarding the extent of derivatization prior to $\mathrm{FAB} / \mathrm{MS}$ analysis. Based on results already cited from ref 41 , as well as a review of the EI/MS data for 16 silylated deoxynucleoside-arylamine adducts [40], the assumption of complete derivatization prior to analysis by FAB/MS may have been incorrect. Nevertheless, the FAB mass spectra in ref 31 are very similar to those we report. For example, a spectrum obtained by using $67 \mathrm{ng}$ of TMS derivatized dG-C8-AF was very similar to Figure 5, obtained by using $5 \mathrm{ng}$. One notable difference is that the extent of hydrolysis is slightly greater in all of the FAB mass spectra reported in ref 31. Vouros's group reported that the bis-TMS protonated molecule was always larger than the trisTMS species under the conditions they described, whereas we often see the opposite. More interesting is the report of better results by using thioglycerol rather than NBA as a FAB matrix.

Derivatization with MSTFA, BSTFA, and MTBSTFA produced similar improvements in sensitivity in our experiments. FAB/MS spectra were readily obtained by using $5 \mathrm{ng}$ of dG-C8-AF derivatized with MTBSTFA (spectrum not shown); however, the abundance of the protonated molecule was relatively small. The tris-(dimethyl, tert-butylsilyl) (DMTBS) derivatized protonated molecule was observed at $m / z 789$ as was a mono-DMTBS $\mathrm{BH}_{2}^{+}$ion $(m / z 445)$ ion attributed to loss of the bis-DMTBS derivatized sugar. The bis-DMTBS protonated molecule $(m / z 675)$ was not observed and the $\mathrm{BH}_{2}^{+}$ion was less intense than either $m / z 789$ or 445 . Numerous other ions in the spectrum were not identified. A particular problem with MTBSTFA derivatization was that for each DMTBS group added, the adduct increased in mass by $114 \mathrm{u}$ rather than the $72 \mathrm{u}$ for TMS. Hence, a DMTBS derivatized nucleoside-carcinogen adduct including a large carcinogen could exceed $1000 \mathrm{u}$ in mass.

A major limitation upon reduction of the sample sizes for mass spectral analysis was dilution of the adducts in the derivatization reaction mixture. The derivatization procedure was changed by omitting the cosolvent to reduce the overall sample volume (Procedure B, Experimental). In subsequent experiments the derivatizing reagent was added directly to the dry nucleoside-adduct residue in a small conical vial. Using this procedure it was possible to derivatize as little as $2 \mathrm{ng}$ of the modified nucleoside with $2 \mu \mathrm{L}$ of MSTFA and to then remove $1 \mu \mathrm{L}$ for analysis. The use of pure MSTFA may also have resulted in a minor reduction in the extent of hydrolysis, as indicated above. This same approach has been proposed in ref 31. In that article the authors recommended derivatization of $200 \mathrm{ng}$ of adduct using 10-20 $\mu \mathrm{L}$ BSTFA [31]. However, they also apparently observed more hydrolysis. We have observed similar results by using other arylamine adducts, namely, $\mathrm{N}$-(deoxyadenosin8-yl)-4-aminobiphenyl (dA-C8-ABP), and N-(deoxyguanosin-8-yl)-2-acetylaminofluorene (dG-C8$A A F)$ (spectra not shown).

\section{Constant Neutral Lass Scans}

The use of CNL scans for the analysis of modified nucleosides was also evaluated. By using this approach all of the ions that arise via a mass loss corresponding to the deoxyribose moiety can then be identified. The characteristic cleavage to produce the $\left[\mathrm{BH}_{2}\right]^{+}$ion has been observed in mass spectra obtained from protonated nucleosides/nucleotides [23-25], carcinogen-modified nucleosides $[26,30,31]$, and silylated carcinogen-modified nucleosides $[27,31]$. We have recently reported the successful application of CNL scans (116 u) to the identification of an unknown carcinogen-nucleoside adduct. When HPLC separated fractions from the in vitro reaction of DNA with a nitro-PAH metabolite, 2-nitropyrene, were examined by using CNL scans, two adducts were identified. One of these was an unusual C8-modified 
deoxyadenosine adduct [30]. By using the same approach, Claeys and co-workers [42] have demonstrated the utility of CNL scans for the analysis of nucleosides of phenyl glycidyl ethers. They reported detection limits of about 1-10 ng. Because daughter ion scans of the protonated molecule were not particularly characteristic of parent ion structures, they used daughters of the $[\mathrm{M}-\mathrm{H}]^{-}$ion, in the negative ion mode, for structure characterization, whereas we employ $\mathrm{BH}_{2}^{+}$ions for the same purpose.

Of particular importance to our study was whether the CNL scan method would allow additional reduction in the minimum amount of sample needed for the analysis of nucleoside-carcinngen adducts by mass spectrometry. As the signals for the protonated molecule and the $\mathrm{BH}_{2}^{+}$ion become weaker at lower concentrations, confirmation of the relationship between these two ions, in unknown samples, becomes increasingly difficult and important. Moreover, any improvement in sensitivity relative to that obtained by using full-scan FAB/MS of MSTFA derivatives would allow detection of adducts at levels approaching 1 pmol.

Underivatized adducts. Underivatized adducts were examined by using CNL scans (116 u) to determine if the observation of parent ions showing loss of the deoxyribose moiety was possible at levels too low to obtain full-scan FAB mass spectra. Whereas full-scan spectra required the analysis of $100 \mathrm{ng}$ of underivatized adducts, the use of these CNL scans allowed adducts to be detected at levels as low as $5 \mathrm{ng}$ (Table 2). Figure 7 shows a FAB CNL mass spectrum obtained by using $5 \mathrm{ng}$ of dG-C8-AF. The protonated molecule at $m / z 447$ is the largest peak in the spectrum. The other signals are attributed to the high noise level at this lower limit of detection. Similar results have been obtained by using $8 \mathrm{ng}$ of $\mathrm{N}$-(deoxyadenosin-8-yl)-4-aminobiphenyl, dA-C8-ABP (spectrum not shown).

Derivatized adducts. Significant reduction in the noise level was observed in CNL scans $(260 \mathrm{u})$ of MSTFA derivatized adducts. With FAB ionization, CNL scans were readily obtained by using $1-5 \mathrm{ng}$ of MSTFA

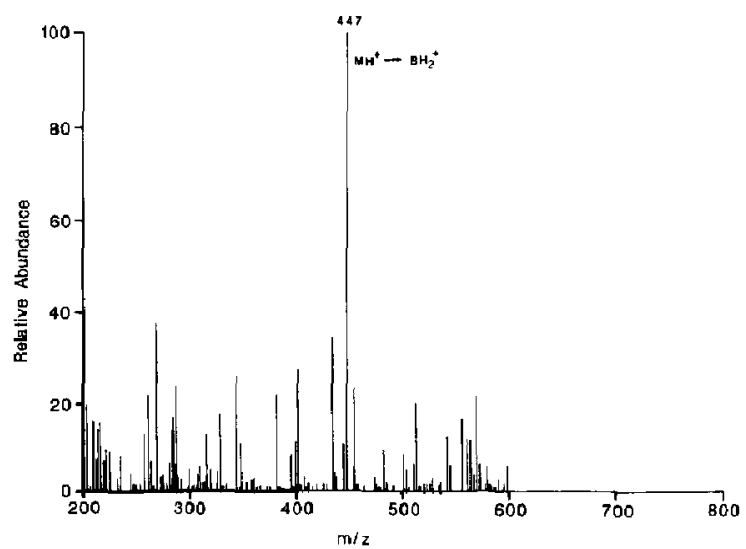

Figure 7. The FAB/CNL spectrum (116 u) obtained by using 5 ng of dG-C8-AF showing the loss of the deoxyribose moiety from the parent ion (protonated molecule) at $m / z 447$.

derivatized dG-C8-AF. Because a derivatized nucleoside was examined, the CNL was $260 \mathrm{u}$ rather than $116 u$, corresponding to the mass of a bis-TMS containing deoxyribose moiety. Typical of these results is the spectrum obtained by using $1 \mathrm{ng}$ dG-C8-AF (Figure 8). The ions at $m / z 591$ and 663 correspond to the protonated molecules of the bis- and tris-TMS derivatives, whereas the ions at $m / z 484$ and 412 are attributed to loss of the AF moiety during derivatization. (The facile cleavage of the carcinogen from TMS derivatives of arylamine-nucleoside adducts, especially C-8 substituted deoxyguanosine derivatives, has been reported elsewhere [40].) These results should be contrasted to those shown in Figure 5, obtained by using $5 \mathrm{ng}$ of MSTFA derivatized adduct via FAB/MS. Satisfactory constant neutral loss scans were consistently obtained by using smaller amounts of sample when the adducts were TMS derivatized. However, the extent of enhancement observed upon TMS derivatization was less with CNL scans (Table 2) than expected based on the results obtained by using FAB/MS (Table 1).

Analysis of unknown adducts. We have already reported the application of CNL scans to the identif-

Table 2. Typical sample sizes needed to obtain FAB CNL spectra for loss of $116 \mathrm{u}$ (the deoxyribose moiety) or $260 \mathrm{u}$ (the bis-TMS derivatized deoxyribose) from the protonated molecule of carcinogen-nucleoside adducts

\begin{tabular}{lcccc}
\hline Adduct & Derivative & $\begin{array}{c}\text { CNL mass } \\
\text { loss }\end{array}$ & Amount $^{\text {a }}$ & Figure $^{-}$ \\
\hline \hline dG-C8-AF & - & 116 & $\mathbf{5} \mathbf{n g}$ & 7 \\
dA-C8-ABP & - & 116 & $8 \mathrm{ng}$ & - \\
dG-C8-AF & MSTFA & 260 & $1 \mathrm{ng}$ & 8 \\
\hline
\end{tabular}

\footnotetext{
Abbreviations: $\mathrm{CNL}=$ constant neutral loss;

FAB = fast atom bombardment ionization:

MSTFA = N-methyl- $\mathrm{N}$-trimethylsilyl-trifluoroacetamide;

$\mathrm{ng}=$ nanograms.

Applied to the probe tip.
} 


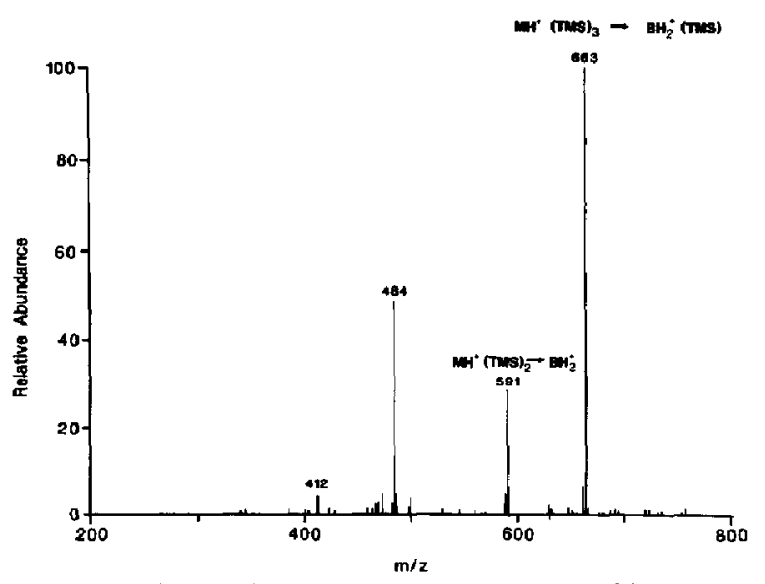

Figure 8. The FAB /CNL spectrum (260 u) obtained by using 1 ng of dG-C8-AF derivatized with MSTFA.

cation of unknown carcinogen-DNA adducts from nitropyrene metabolites [30]. Another recent experiment also demonstrates this application. Several isolated HPLC fractions, some containing carcinogenmodified DNA, were submitted to the laboratory for identification. Prior to mass spectral analysis the DNA was reacted with the N-hydroxy derivative of the heterocyclic aromatic amine 2-amino-1-methyl-6phenylimidazo[4,5-b]pyridine (PHiP), the modified DNA was hydrolyzed to the nucleosides, and HPLC fractions containing UV adsorbing peaks with retention times consistent with modified nucleosides were collected. The individual fractions were dried, TMS derivatized, and submitted to the laboratory as unknowns. The CNL scan (260 u) obtained from one of the fractions is shown in Figure 9. The intense ion at $m / z 706$ corresponds to the tris-TMS derivative of dG-C8-PHiP. Additional evidence for this assignment was obtained by comparison of daughter ions from the $\mathrm{BH}_{2}^{+}$ion $(m / z$ 374) and synthetic G-C8-PHiP.

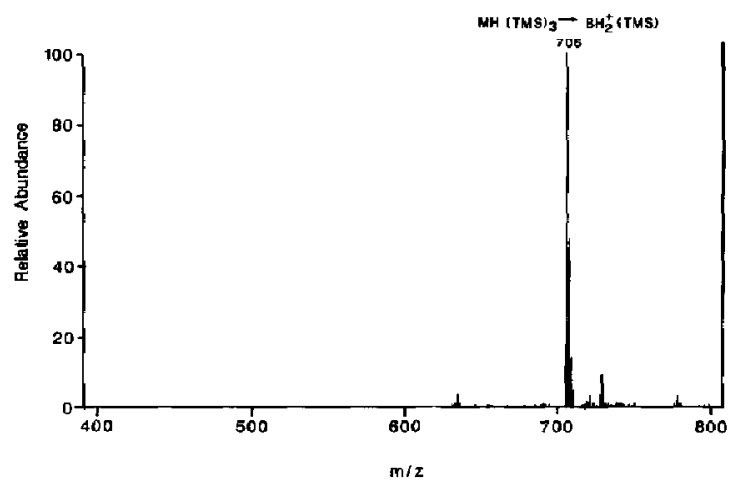

Figure 9. The FAB CNL spectrum (260 u) of an unknown HPLC separated component from the hydrolysate of N-hydroxy-PHil' treated DNA.

\section{Daughter Ion Scans}

The use of daughter ion scans to provide evidence regarding nucleoside-adduct structures was also investigated. For unknowns, even modest details regarding adduct structure could be extremely useful. For example, identification of a specific base would allow the weight of the carcinogen to be deduced. The MS/MS methodology is widely accepted as a means of detecting trace components in complex mixtures [43]. Vouros and co-workers [31] have developed a method for the detection of specific carcinogen-DNA adducts a the $100 \mathrm{ng}$ level based on TMS derivatization, FAB/MS analysis, and, finally, B/E linked scans. Our method differs primarily based on the use of a CNL scan rather than FAB/MS in the first step of mass spectral analysis. These CNL scans simplify the identification of the parent ions that are used in the second step of identification based on daughter ion scans. We also note some differences in daughter ions obtained by using a triple quadrupole mass spectrometer and low energy collisional activation (CA), described below.

The typical procedure for characterizing analyte structures via soft ionization and MS/MS is CA of the analyte protonated molecule. For underivatized arylamine-nucleoside adducts this produces an intense $\mathrm{BH}_{2}^{+}$ion. For example, the daughter ion spectrum obtained when the protonated molecule $(\mathrm{m} / \mathrm{z} 447)$ of dG-C8-AF was mass selected and collisionally activated showed a single major fragment ion at $m / z 331$ (spectrum not shown). Similar results were observed with the other model arylamine-nucleoside adducts. This fragment ion provides no additional evidence regarding the adduct's structure beyond that also observed in the FAB/MS and FAB CNL scans.

With derivatized adducts the results were similar. The daughter ions of the mono-TMS derivative of dG-C8-AF included a $\mathrm{BH}_{2}^{+}$ion at $m / z 331$ and a TMS-BH ${ }_{2}^{+}$ion $72 \mathrm{u}$ higher in mass at $m / z$ 403. This spectrum (Figure 10) illustrates two important points. First, as with underivatized nucleoside-adducts,

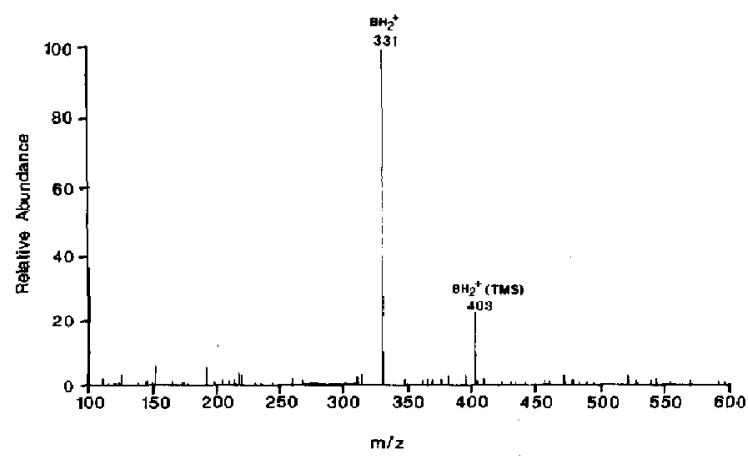

Figure 10. The FAB daughter ion spectrum from the protonated molecule $(m / z 519)$ of the mono-TMS derivative of dGC8-AF. 
daughter ions from the protonated molecule do not provide additional data regarding adduct substructure. Second, derivatization complicates interpretation when an adduct containing TMS groups may include more than one isomer. The ion at $m / z 403$ in Figure 10 must have arisen via the loss of an underivatized sugar from a protonated molecule having the TMS group attached to the base or the AF moiety. On the other hand, the ion at $\mathrm{m} / \mathrm{z} 331$ must have arisen from a protonated molecule having a TMS group on the sugar but not the base or carcinogen moiety.

A simple strategy was developed to avoid problems associated with the formation of isomers accompanying the TMS derivatization step. Derivatized adducts were used to obtain CNL (260 u) and daughter ion spectra; but the specific daughter ions were underivatized $\mathrm{BH}_{2}^{+}$fragments rather than TMS-containing protonated molecules. The incomplete derivatization of the carcinogen/base moiety and the loss of one TMS in the FAB matrix liquid have resulted in the observation of non-TMS containing $\mathrm{BH}_{2}^{+}$ions from all of the MSTFA dcrivatized carcinogen-nucleoside adducts examined in our laboratory. Fortuitously, for carcinogen-deoxynucleoside adducts, the $\mathrm{BH}_{2}^{+}$ions show considerably more structurally characteristic fragmentation than protonated molecules [31]. Be- cause the carcinogen is invariably bound to the base rather than the deoxyribose moiety in carcinogenDNA adducts, this strategy should not result in loss of structure specificity. The observation that daughter ion spectra from several protonated G-C8-adducts are indistinguishable from the daughters of the corresponding $\mathrm{BH}_{2}^{+}$fragments supports this hypothesis. For example, the daughters of the $\mathrm{BH}_{2}^{+}$fragment ion of $\mathrm{dG}-\mathrm{C} 8 \mathrm{ABP}$ and the protonated molecule of the corresponding carcinogen base adduct (N-(guan-8-yl)4-aminobiphenyl) are compared in Table 3 .

Table 3 shows the masses of significant daughter ions observed from the $\mathrm{BH}_{2}^{+}$of dG-C8-ABP. The frequency of occurrence of daughters using various collision energies is also reported. Subjective review of these data, and the individual daughter ion spectra used to prepare Table 3 , suggested that although there was significant variability between spectra at all collision energies, $50-\mathrm{eV}$ collisions generally produced more abundant and reproducible fragmentation. To provide consistency this value was subsequently used in the remaining daughter ion experiments.

A daughter ion spectrum obtained by using the $\mathrm{BH}_{2}^{+}$ion from $20 \mathrm{ng}$ of dG-C8-AF derivatized by using MSTFA is shown in Figure 11. These results are compared in Table 4 with daughters oblained by using dG-C8-ABP and are shown in Table 3. Peaks

Table 3. The frequency of observation of selected daughter ions from the $\mathrm{BH}_{2}^{+}$ion of $\mathrm{dG}-\mathrm{C} 8 \mathrm{ABP}$ and a comparison with daughter ions from the protonated molecule of synthetic G-C8-ABPa

\begin{tabular}{|c|c|c|c|c|c|c|}
\hline \multirow{2}{*}{$\begin{array}{l}\text { lan } \\
\text { mass }\end{array}$} & \multirow{2}{*}{$\begin{array}{c}\text { Mass } \\
\text { loss }\end{array}$} & \multicolumn{5}{|c|}{ Experiment Number } \\
\hline & & 1 & 2 & 3 & $2^{\prime b}$ & $4^{c}$ \\
\hline 302 & 17 & + & + & + & + & + \\
\hline 277 & 42 & $3 / 4$ & + & $2 / 3$ & + & + \\
\hline 276 & 43 & $2 / 4$ & $2 / 6$ & - & - & - \\
\hline 275 & 44 & - & $2 / 6$ & - & - & + \\
\hline 249 & 70 & $2 / 4$ & + & $1 / 3$ & + & + \\
\hline 247 & 72 & $3 / 4$ & $5 / 6$ & - & + & + \\
\hline 208 & 111 & + & + & + & + & + \\
\hline 207 & 112 & + & $5 / 6$ & + & + & + \\
\hline 195 & 124 & + & + & $2 / 3$ & + & + \\
\hline 180 & 139 & + & + & + & + & + \\
\hline 168 & 151 & - & $5 / 6$ & + & + & + \\
\hline 165 & 154 & + & + & $1 / 3$ & + & + \\
\hline 153 & 166 & $2 / 4$ & + & + & + & + \\
\hline 140 & 179 & + & + & + & + & + \\
\hline 123 & 196 & + & + & + & + & + \\
\hline 112 & 207 & + & + & + & + & + \\
\hline 105 & 214 & - & $3 / 6$ & - & - & - \\
\hline 95 & 224 & $1 / 4$ & + & $2 / 3$ & + & + \\
\hline \multicolumn{7}{|l|}{ Collision } \\
\hline energy $(e V)^{d}$ & & 30 & 50 & 90 & 50 & 50 \\
\hline
\end{tabular}

"A " +" symbol indicates that daughter ion was detected in all experiments. A fraction indicates the frequency with which a daughter ion was observed.

$\mathrm{b}$ The daughter ions expected mare than $50 \%$ of the time from a single analysis at $50 \mathrm{gV}$

c The daughter ions observed fram the protonated molecule of G-8-ABP, for comparison.

$\mathrm{deV}=$ electron volts. the laboratory value for collision energy. 


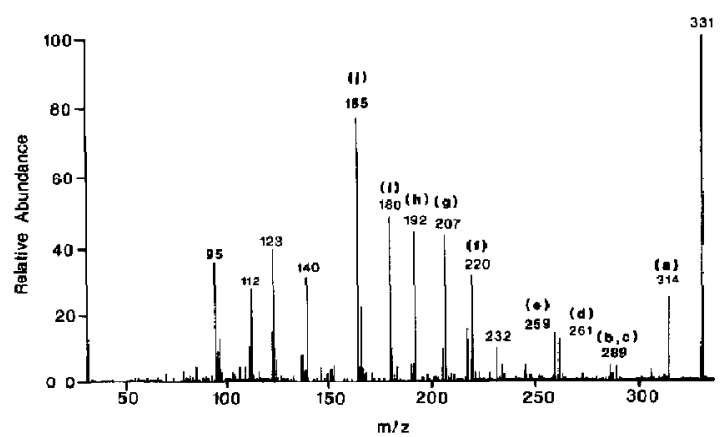

Figure 11. The $\mathrm{FAB}$ daughter ion spectrum from the $\mathrm{BH}_{2}^{+}$ fragment ion obtained by using $20 \mathrm{ng}$ of $\mathrm{dG}-\mathrm{C} 8-\mathrm{AF}$ derivatized with MSTFA.

labeled a to $j$ occurred via the same neutral losses for both of these C-8 substituted deoxy-guanosine adducts. This is attributed to fragmentation in which the carcinogen is retained within the part of the molecule having the positive charge. Experiments using a deuterated adduct (dG-C $8-\mathrm{ABP}_{\mathrm{d}} \mathrm{d}_{9}$ ) confirmed retention of the carcinogen moiety in these fragments. Daughter ions including the carcinogen moiety retained eight or nine of the nine deuterium atoms originally bound to the biphenyl group in the ABP moiety of the deuterated adduct.
Other characteristic ions showed the same masses rather than identical neutral losses. The ions at $m / z$ 95, 112, 123, and 140, observed in the spectra of $\mathrm{dG}-\mathrm{C} 8-\mathrm{AF}$ and $\mathrm{dG}-\mathrm{C} 8-\mathrm{ABP}$, are attributed to fragments from which the carcinogen moiety has been lost. Because the ions a to j resulted from retention of the carcinogen moiety, ions resulting from these specific neutral losses should be observed from the $\mathrm{BH}_{2}^{+}$ ions of all C8-dG adducts. Similarly, the ions showing loss of the carcinogen should have common masses $(m / z$ 95, 112, 123, and 140) for all C8-dG adducts. An exception is that because fragment $j$ occurs via cleavage within the carcinogen, it may be specific for $\mathrm{CB}$ adducts linked to the carcinogen via an amine moiety (i.e., arylamines).

The specific cleavages observed in the daughter ion spectrum (see Figure 11) are shown in Scheme I. These cleavages were chosen based on the hypothesis that for low energy collisions, the even electron $\mathrm{BH}_{2}^{+}$ fragment ion would not fragment via loss of radicals. A previous report of radical ions in the FAB mass spectra of TMS derivatives of related compounds was attributed to the presence of an Si atom in the TMS moiety [33]. Because no TMS groups are present on the $\mathrm{BH}_{2}^{+}$ions used as parent ions, radical ions would not be expected. The correspondence between the spectra for the two dG-C8 adducts, as well as the

Table 4. Comparison of the major daughter ions in the tandem mass spectra of dG-C8-ABP. $\mathrm{dG}-\mathrm{C} 8-\mathrm{AF}$, and $\mathrm{dA}-\mathrm{C} 8-\mathrm{ABP}$ showing common neutral losses or common fragment masses ${ }^{\mathrm{a}}$

\begin{tabular}{|c|c|c|c|c|}
\hline Mass loss & dG-C8-ABP & dG-C8-AF & Fragment $^{\circ}$ & dA-C8-ABP \\
\hline 17 & + & + & $\mathbf{a}$ & + \\
\hline 42 & + & + & $\mathbf{b}$ & \\
\hline 44 & $(2 / 6)$ & + & $c$ & + \\
\hline 70 & + & + & d & \\
\hline 72 & $(5 / 6)$ & + & a & \\
\hline 111 & + & + & $f$ & \\
\hline 112 & + & + & & \\
\hline 124 & + & $+d$ & $\mathrm{~g}$ & \\
\hline 139 & + & + & h & \\
\hline 151 & + & $++^{e}$ & $\mathrm{i}$ & \\
\hline 166 & + & + & $\cdot \mathbf{j}$ & \\
\hline \multicolumn{5}{|l|}{ Mass } \\
\hline 207 & + & $+a$ & & + \\
\hline 195 & + & & $\mathrm{g}$ & + \\
\hline 180 & + & $+{ }^{e}$ & h & + \\
\hline 168 & + & & i & + \\
\hline 153 & + & & j & + \\
\hline 140 & + & + & & \\
\hline 123 & + & + & & \\
\hline 112 & + & + & & \\
\hline 105 & + & & & \\
\hline 95 & + & + & & \\
\hline
\end{tabular}

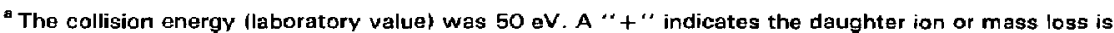
observed.

"A " $2 / 6$ " indicates that this daughter ion was observed in only two of six replicate analyses.

"This term refers to proposed structures for the daughter ions labeled in Figure 11 as shown in Scheme I.

This fragment at $\mathrm{m} / 2207$ is attributed to loss of $124 \mathrm{u}$ via cleavage $\mathrm{g}$ in Scheme $\mathrm{I}$.

${ }^{8}$ This fragment at $m / 2180$ is attributed to loss of 151 u via cleavage i in Scheme I.
} 


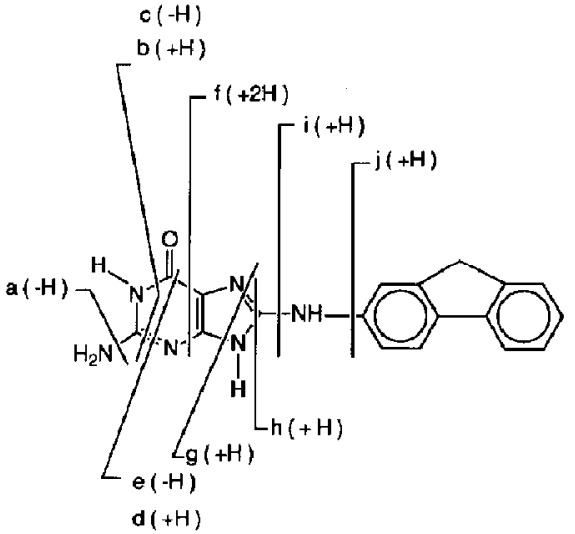

Scheme 1. Proposed fragmentation of the $\mathrm{BH}_{2}^{+}$ion from $\mathrm{dG}$ Cy-AF corresponding to peaks labeled a to $\mathrm{j}$ in Figure 11 and Table 4.

ready assignment of the carcinogen containing fragments from the adduct having deuterium isotopes on the aromatic hydrogens of the carcinogen moiety, provide strong evidence for the assignments in Scheme I. However, even if these include errors, the daughter ion methodology can be used for structure characterization based on the empirically observed correlation between neutral losses and fragment ion Inasses with the andlyte structures.

A comparison was made with a model adduct of a different nucleoside having the same carcinogen and similar carcinogen-base bonding (dA-C8-ABP). The daughter ion spectrum (not shown) was obtained by using $42 \mathrm{ng}$ of adduct. Most of the dG characteristic losses associated with retention of the carcinogen moiety in the neutral fragment were not observed with the dA adduct (see Table 4). Exceptions are ions labeled $\mathrm{a}$ and $\mathrm{c}$. These losses, $\mathrm{NH}_{3}$ and $\mathrm{C}_{2} \mathrm{~N}_{2} \mathrm{H}_{2}$, are relatively small molecules and might be expected from either $\mathrm{dA}$ or $\mathrm{dG}$ adducts. However, some ions are common to both the ABP adducts. For example, ions labeled $g, h, i$, and $j$ are attributed to loss of most of the base moiety. Any remaining portion of the base substructure is identical for $\mathrm{dG}-\mathrm{C} 8-\mathrm{ABP}$ and $\mathrm{dA}-\mathrm{C} 8$ $\mathrm{ABP}$, as shown in Scheme 1 .

Some confusion may arise because of the coincidence of daughter ion masses attributed to different fragmentation pathways in two of the adducts. The ions at $\mathrm{m} / z 207$ and 180 for dG-C8-AF and dG-C8$A B P$ are not attributed to the same structures (see Table 4). The ions at $m / z 207$ and 180 in dG-C8-AF correspond to loss of 124 and $151 \mathrm{u}$, respectively, from the $\mathrm{BH}_{2}^{+}$. Hence, the $m / z 207$ and 180 ions from dG-C8-AF and the $m / z 168$ and 195 ions from dG-C8$A B P$ would be attributed to the same cleavage within the base moiety. The $m / z 180$ from dG-C8-AF arises from loss of the $d G$ moiety to give $\left[\mathrm{C}_{13} \mathrm{H}_{10} \mathrm{~N}\right]^{+}$.

These results should be contrasted to those reported in ref 31. Daughter ions from the $\mathrm{BH}_{2}^{+}$ion from dG-C8-AF were examined by using high energy collisional activation and $\mathrm{B} / \mathrm{E}$ scans. Significant daughter ions were reported at $m / z$ 314, 284, 259, $232,206,191,180$, and 165 . The ions at $m / z$ 314, 259, 232, 180, and 165 are prominent in Figure 11 as well. These ions, common to both studies, were attributed in ref 31 to the same structures we propose in Scheme I. The ions at $m / z 284,206$, and 191 were observed in high energy, but not low energy, collisions. The ions at $m / z 206$ and 191 could be radical fragments resulting from high energy collisions, or they could be misassigned in the $\mathrm{B} / \mathrm{E}$ scan. The authors of ref 31 pointed out that an error in their daughter ion mass assignments of $1 \mathrm{u}$ was possible because of the use of linked scans. Because we have observed $\mathrm{m} / z 207$ and 192 in numerous daughter ion scans from dG-C8-AF, and this same mass loss (of 124 and $151 \mathrm{u}$ ) from $\mathrm{dG}-\mathrm{C} 8-\mathrm{ABP}$, and $\mathrm{dG}-\mathrm{C} 8-\mathrm{AF}$, under a variety of experimental conditions, we attribute this difference to an error in the linked scan derived daughter ion mass assignments. The other ion observed in ref 31 , at $\mathrm{m} / z$ 284, was not observed in our experiments, but was also attributed to an even electron fragment. These results suggest daughter ion spectra obtained by using low or high energy collisions provide sufficient structure specificity for use in characterizing adduct structures, even when the experiments are performed in different laboratories. Furthermore, with the exception of the two daughter ions in the $\mathrm{B} / \mathrm{E}$ scans that can be attributed to an error in mass assignment, all of the daughter ions from the $\mathrm{BH}_{2}^{+}$ions of several adducts, in both studies, can be attributed to even electron fragmentation.

\section{Conclusion}

Our methodology combines three experimental approaches to the characterization of low levels of carcinogen-deoxynucleoside adducts. The utility of each component approach for the analysis of these or related compounds by mass spectrometery has been previously reported. The method incorporates the use of CNL scans for screening [30, 42], TMS derivatization for sensitivity enhancement [31-33], and daughters of the $\mathrm{BH}_{2}^{+}$ion for structure characterization [31].

FAB ionization with CNL scans of MSTFA derivatized samples allowed identification of carcinogen-deoxynucleoside adducts at levels as low as $1 \mathrm{ng}$. For a carcinogen-nucleoside adduct with a molecular weight of $500 \mathrm{u}$, this corresponds to about 2 pmol. With this detection limit the analysis of in vivo samples from animals dosed with carcinogens should be possible based on one adduct per $10^{6}$ nucleotides (manuscript in preparation).

Daughter ion scans were used to differentiate $C-8$ substituted arylamine adducts of deoxyadenosine and deoxyguanosine by using the $\mathrm{BH}_{2}^{+}$ion as the parent. For dG-C8-ABP this fragment ion showed the same daughter ion spectrum as the protonated molecule of 
the carcinogen-base, G-C8-ABP. Fragmentation of the $\mathrm{BH}_{2}^{+}$parent ions was largely in agreement with previously reported results obtained by using high energy collisions.

\section{Acknowledgments}

N-(deoxyadenosin-8-yl)-4-aminobiphenyl, and HPLC fractions from the reaction of $\mathrm{N}$-hydroxy-PHiP with DNA were kindly provided by Dr. F. F. Kadlubar of the National Center for Toxicological Research (NCTR). N-(deoxyguanosin-8-yl)-2aminofluorene and N-(deoxyguanosin-8-yl)-2-acetylaminofluorene were gifts of Dr. F. A. Beland (NCTR), who also provided advice, consultation, and encouragement.

This work was supported in part by The Center for Indoor Air Research (CIAR \#90-002).

\section{References}

1. Beland, F. A.; Poirier, M. C. In The Pathabiology of Neoplasia; A. E. Sirca, Ed.; Plenum: New York, 1989; pp 57-80.

2. Perera, F. Environmental Health Perspectioes 1987, 76, 141.

3. Harris, C. C.; Weston, A.; Willey, J. C.; Trivers, G. E.; Mann, D. L. Environmental Health Perspectives 1987, 75, 109-119.

4. de Serres, F. J. Mutation Research 1988, 203, 55-68.

5. Perera, F. P. Mutation Research 1988, 205, 255-269.

6. Perera, F. P.; Weinstein, I. B. J. Chrontic Dis. 1981, 35, $581-600$.

7. Perera, F. P. I. Natl. Cancer Inst. 1987, 78, 887-898.

8. Everson, R. B.; Randerath, E.; Santella, R. M.; Cefalo, R. C.; Avitts, T. A.; Randerath, K. Science 1986, 231, 54-57.

9. Perera, F. P.; Poirier, M. C.; Yuspa, S. H.; Nakayama, J.; Jaretzki, A.; Curnen, M. C.; Knowles, D. M.; Weinstein, I. B. Carcinogenesis 1982, 3, 1405-1410.

10. Haugen, A.; Becher, G.; Benestad, C.; Vahakangas, K. Trivers, G. E.; Newman, M. J.; Harris, C. C. Concer Res. 1986, 46, 4178-4183.

11. Harris, C. C; Vahakangas, K.; Newman, M.; Trivers, G. E.; Shamsuddin, A.; Sinopoli, N.; Mann, D. L.; Wright, W. E. Proc. Natl. Acad. Sci. USA 1985, 82, 6672-6676.

12. Shamsuddin, A. K. M.; Sinopoli, N. T.; Hemminki, K.; Boesch, R. R.; Harris, C. C. Cancer Res. 1985, 45, 66-68.

13. Umbenhauer, D.; Wild, C. P.; Montesano, R.; Saffhill, R.; Boyle, J, M.; Huh, N.; Kirstein. U.; Thomale, J.; Rajewsky, M. F.; Lu, S. H. Int. I. Cancer 1985, 36, 661-665.

14. Vahakangas, K.; Haugen, A.; Harris, C. Carcinogenesis 1985, 6, 1109-1116.

15. Kanderath, E.; Avitts, T. A.; Reddy, V.; Miller, R. H.; Everson, R. B,; Randerath, K. Cancer Res. 1986, 46, 5869-5877.

16. Dunn, B. P.; Stich, H. F. Carcinogenesis 1986, 7, 1115-1120.

17. Phillips, D. H.; Hewer, A.; Grover, P. L. Carcinogenesis 1986, 7, 2071-2075.

18. Gupta, R. C,; Reddy, M. V.; Randerath, K. Carcinogenesis 1982, 3, 1081-1092.
19. Poirier, M. C. Environ. Mutagern. 1984, 6, 879-887.

20. Minnetian, O.; Saha, M.; Giese, R. W. J. Chromatogr. 1987, $410,453-457$

21. Trainor, T. M.; Giese, R. W.; Vouros, P. T. Chromatogr. $1988,452,369-376$.

22. Fedtke, N.; Boucheron, J. A.; Turner, M. J. Jr.; Swenberg, J. A. Carcinogenesis 1990, 11, 1279-1285.

23. Schram, K. H. Trends in Analytical Chemistry 1988, 7, 28-32.

24. Moser, H.; Wood, G. W. Biomed. Environ. Mass Spectrom. $1988,15,547-551$.

25. Crow, F. W.; Tomer, K. B.; Grass, M. L.; McCloskey, J. A.; Bergstrom, D. E. Analytical Biochemistry 1984, 139, 243-262.

26. Mitchum, R. K.; Evans, F. E.; Freeman, J. P.; Roach, D. Int. I. Mass Spectrom. Ion Phys. 1983, 46, 383-386.

27. Silk, N. A.; Lay, J. O. Jr.; Martin, C. N. Biochemical Pharmacology 1989, 38, 279-285.

28. Lasko, D. D.; Basu, A. K.; Kadlubar, F. F.; Evans, F. E.; Lay, J. O. Jr.; Essigmann, J. M. Biochemistry 1987, 26, 3072-3081.

29. Delclos, K. B.; Miller, D. W.; Lay, J. O. Jr.; Casciano, D. A.; Walker, R. P.; Fu, P. P.; Kadlubar, F. F. Carcinogenesis 1987, 8, 1703.

30. Fu, P. P.; Miller, D. W.; Von Tungeln, L. S.; Bryant, M. S.; Lay, J. O. Jr.; Huang, K.; Jones, L.; Evans, F. E. Corcinogenesis 1991, 12, 609-616.

31. Annan, R. S.; Giese, R. W.; Vouros, P. Anal. Biochem. 1990, 191, 86-95.

32. Weng, Q.-M.; Hammargren, W. M.; Slowikowski, D.; Schram, K. H.; Borysko, K. Z.; Wotring, L. L.; Townsend, L. B. Analytical Biochemistry 1989, 178, 102-106.

33. Schram, K. H.; Slowikowski, D. L. Biomed. Etrviron. Mass Spectrom. 1986, 13, 263-264.

34. Mohamed, G. B.; Nazareth, A.; Hayes, M. J.; Giese, R. W.; Vouros, P. I. Chromatogr. 1984, 314, 211-217.

35. Nazareth, A.; Joppich, M.; Abdel-Baky, S.; O'Connel, K.; Sentissi, A.; Giese, R. W. 1. Chromatogr. 1984, 314, 201-210.

36. Bryant, M. S.; Getek, T. A.; Korfmacher, W. A.; Kadlubar, F. F.: Lay, J. O. Jr. Second International Symposium on Mass Spectrometry and the Health and Life Sciences, San Francisco, CA, 1989.

37. Bryant, M. S.; Freeman, J. P.; Lay, J. O. Jr. Proceedings of the 38th ASMS Conference on Mass Spectrometry and Allied Topics, Tucson, AZ, 1990; pp. 962-963.

38. Bryant, M. S.; Lay, J. O. Jr. The American Association for Cancer Research, Washington, DC, 1990.

39. Lee, M.; King. C. M. Chem,-Biol. Int. 1981, 34, 239-48.

40. Mitchum, R. K.; Freeman, J. P.; Beland, F. A.; Kadlubar, F. F. Mass Spectrometry in the Health and Life Sciences; Burlingame, N. L. and Castagnoli, N. Jr., Eds.; Elsevier Science Publishers: Amsterdam, The Netherlands, 1985; pp 547-580.

41. Beland, F. A.; Tullis, D. L.; Kadlubar, F. F.; Straub, K. M.; Evans, F. E. Chem.-Biol. Int. 1980, 31, 117.

42. Claereboudt, J.; Esmans, E. L.; Van den Eeckhout, E. G.; Claeys, M. Nucleosides and Nucleotides 1990, 9, 333-344.

43. Johnson, J. V.; Yost, R. A. Aral. Chem. 1985, 57, 758A-768A. 